\title{
A new Arctic hadrosaurid from the Prince Creek Formation (lower Maastrichtian) of northern Alaska
}

Hirotsugu Mori, Patrick S. Druckenmiller, and Gregory M. Erickson

Acta Palaeontologica Polonica 61 (1), 2016: 15-32 doi:http://dx.doi.org/10.4202/app.00152.2015

The Liscomb bonebed in the Price Creek Formation of northern Alaska has produced thousands of individual bones of a saurolophine hadrosaurid similar to Edmontosaurus; however, the specific identity of this taxon has been unclear, in part because the vast majority of the remains represent immature individuals. In this study, we address the taxonomic status of the Alaskan material through a comparative and quantitative morphological analysis of juvenile as well several near adult-sized specimens with particular reference to the two known species of Edmontosaurus, as well as a cladistic analysis using two different matrices for Hadrosauroidea. In the comparative morphological analysis, we introduce a quantitative method using bivariate plots to address ontogenetic variation. Our comparative anatomical analysis reveals that the Alaskan saurolophine possesses a unique suite of characters that distinguishes it from Edmontosaurus , including a premaxillary circumnarial ridge that projects posterolaterally without a premaxillary vestibular promontory, a shallow groove lateral to the posterodorsal premaxillary foramen, a relatively narrow jugal process of the postorbital lacking a postorbital pocket, a relatively tall maxilla, a relatively gracile jugal, a more strongly angled posterior margin of the anterior process of the jugal, wide lateral exposure of the quadratojugal, and a short symphyseal process of the dentary. The cladistic analyses consistently recover the Alaskan saurolophine as the sister taxon to Edmontosaurus annectens + Edmontosaurus regalis. This phylogenetic assessment is robust even when accounting for ontogenetically variable characters. Based on these results, we erect a new taxon, Ugrunaaluk kuukpikensis gen. et sp. nov. that contributes to growing evidence for a distinct, early Maastrichtian Arctic dinosaur community that existed at the northernmost extent of Laramidia during the Late Cretaceous.

Key words: Dinosauria, Hadrosauridae, Saurolophinae, Edmontosaurini, ontogeny, Cretaceous, Prince Creek Formation, Arctic, Alaska.

Hirotsugu Mori [hmori@alaska.edu], Department of Geosciences, University of Alaska Fairbanks, 900 Yukon Drive, Fairbanks, AK 99775-5780, USA; University of Alaska Museum, 907 Yukon Drive, 
Fairbanks, AK 99775, USA; and Saikai City Board of Education;

Setoitanoura Go, Oseto Cho, Saikai City, Nagasaki Prefecture, 857-2301, Japan. Patrick S. Druckenmiller [psdruckenmiller@alaska.edu], University of Alaska Museum, 907 Yukon Drive, Fairbanks, AK 99775, USA; and Department of Geosciences, University of Alaska Fairbanks, 900 Yukon Drive, Fairbanks, AK 99775-5780, USA. Gregory M. Erickson [gerickson@bio.fsu.edu], Department of Biological Science, Florida State University, 319 Stadi-um Drive, Tallahassee, FL 32306-4295, USA.

This is an open-access article distributed under the terms of the Creative Commons Attribution License (for details please see creativecommons.org), which permits unrestricted use, distribution, and reproduction in any medium, provided the original author and source are credited.

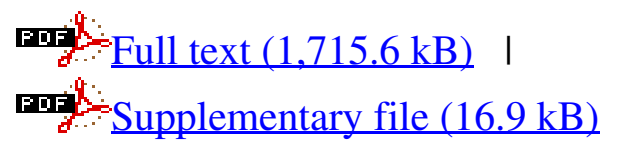

